Copyright (C) 2013 IEEE. Personal use of this material is permitted. Permission from IEEE must be obtained for all other uses, in any current or future media, including reprinting/republishing this material for advertising or promotional purposes, creating new collective works, for resale or redistribution to servers or lists, or reuse of any copyrighted component of this work in other works. 


\title{
First Results from AAVS 0.5: A Prototype Array for Next- Generation Radio Astronomy
}

\author{
P. J. Hall ${ }^{1}$, A. T. Sutinjo ${ }^{1}$, E. de Lera Acedo ${ }^{2}$, R. B. Wayth ${ }^{1}$, N. Razavi-Ghods ${ }^{2}$, T. M. \\ Colegate $^{1}$, A. J. Faulkner ${ }^{2}$, B. Juswardy ${ }^{1}$, B. Fiorelli ${ }^{3}$, T. Booler ${ }^{1}$, J. G. Bij de Vaate ${ }^{3}$, M. \\ Waterson $^{1}$ and S. J. Tingay ${ }^{1}$
}

\begin{abstract}
This paper provides an overview of the Aperture Array Verification System 0.5 (AAVS 0.5), co-located and operated in conjunction with the Murchison Widefield Array (MWA) near the Australian SKA core site. AAVS 0.5 is based on log-periodic antennas of a type potentially useful in nextgeneration low-frequency arrays such as SKA-low. We report on our progress by discussing results obtained thus far as well as test plans for the near future. A number of lessons learned will be presented, demonstrating that hands-on experience constitutes an essential knowledge-base in the pre-construction phase of a radio-telescope such as the SKA.
\end{abstract}

\section{INTRODUCTION}

Verification systems are essential components of the current pre-construction phase of the Square Kilometre Array (SKA) radio telescope [1], demonstrating that candidate designs meet functionality, cost and site requirements targets. The concept design for the low-frequency band of the telescope (SKA-low) calls for sparse, phased aperture arrays of wideband antennas spanning, at the time of writing, 50-350 MHz [1] (formerly, 70-450 MHz [2]). To demonstrate and evaluate potential telescope design solutions, a series of Aperture Array Verification Systems (AAVSs) are being constructed sequentially. AAVS 0.5 is an array of $16 \log$-periodic dipole "SKALA" antennas [3] of a type being evaluated for SKA-low. It is deployed at the Murchison Radioastronomy Observatory (MRO) in remote Western Australia, near the proposed Australian SKA core site.

We describe AAVS 0.5 and report first results from a number of tests in Sections 2 and 3. Sections 4 and 5 describe test plans for the near-future and summarize the lessons learned, respectively.

\section{AAVS 0.5 OVERVIEW}

AAVSs are an initiative of the Aperture Array Design and Construction (AADC) Consortium, with AAVS 0.5 being constructed by ASTRON, ICRAR, and University of Cambridge to gain direct experience in prototyping systems near the Australian SKA core site. AAVS 0.5 is the first system constructed in Australia; it is preceded by AAVS 0 [4], an identical array constructed in Lord's Bridge (UK) and, subject to site approval processes, it will be succeeded by AAVS 1, an array comparable in size to an SKA-low "station" [1].

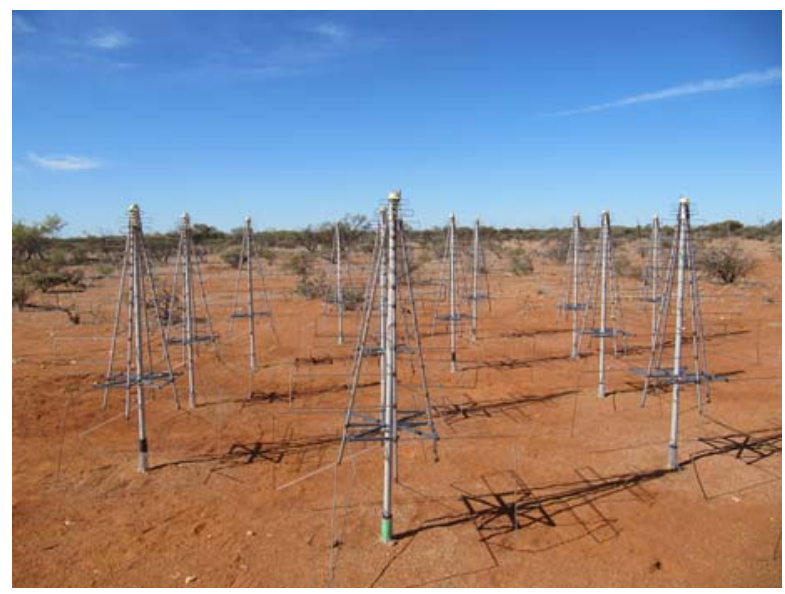

Figure 1: A photograph of AAVS 0.5 array as deployed at the MRO in November 2012.

Construction and testing of AAVS 0.5 is undertaken in close collaboration with the MWA [5, 6] under that telescope's external instruments policy. Situated amongst the MWA tiles, this arrangement affords AAVS 0.5 access to infrastructure such as power, signal conditioning and data processing. As we will touch on later, it also enables characterization of AAVS 0.5 via interferometric measurements with the MWA; these outcomes will inform subsequent AAVSs and possible upgrade paths for the MWA.

\subsection{Technical description}

AAVS 0.5 consists of 16 dual-polarized SKALA antennas (Fig. 1) designed to operate from 70 to $450 \mathrm{MHz}$. As seen in Fig. 2, the elements are arranged pseudo-randomly in an $8 \mathrm{~m}$ diameter circle. This configuration has been selected to facilitate wideband array beamforming by mitigating grating lobe and scan blindness problems typically found in regularly spaced arrays [7, 8].

As an engineering test-bed, the array is flexible when it comes to signal conditioning and data processing. However, as opposed to uncritically borrowing a "ready-made" receiving system from elsewhere, we develop a practice of first capturing

\footnotetext{
${ }^{1}$ International Centre for Radio Astronomy Research (ICRAR), Curtin University, GPO Box U1987, Perth, WA 6845, Australia; e-mail: peter.hall@icrar.org, tel.: +61(0)892669899, fax: +61(0)892669246.

${ }^{2}$ Cavendish Laboratory, University of Cambridge, JJ Thompson Avenue, Cambridge, CB3 0HE UK, e-mail:eloy@mrao.cam.ac.uk, tel.: +44 (0)1223 337294, fax: +44 (0)1223 354599.

${ }^{3}$ ASTRON, Netherlands Institute for Radio Astronomy, 7990 AA, Dwingeloo, The Netherlands, e-mail: fiorelli@anstron.nl, tel: + 31 (0)

521595 100, fax: +31 (0) 521595101
} 
system specifications based on the requirements for a test with the aim to foster a disciplined verification and validation approach. We will illustrate this process with the measurements we have performed so far.

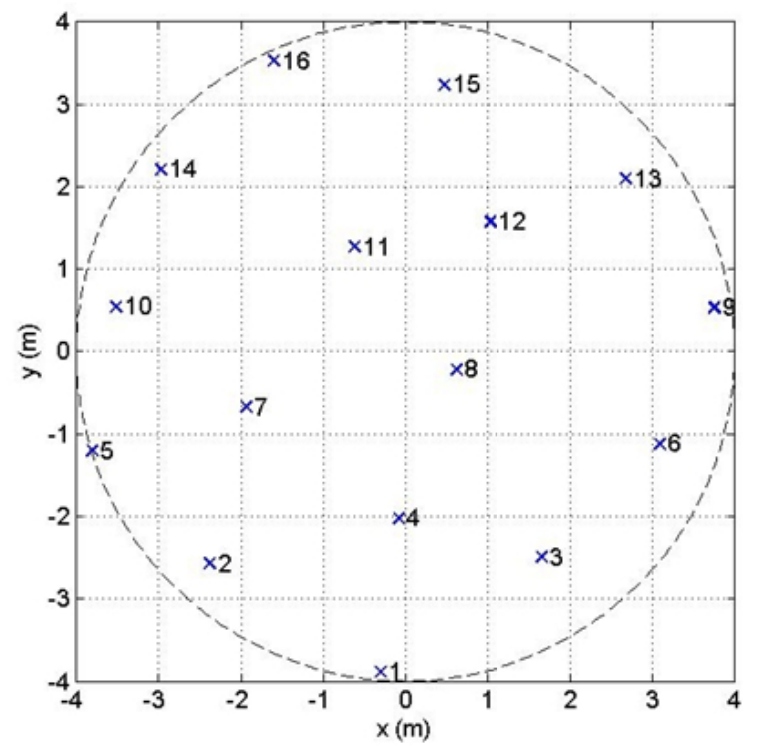

Figure 2: AAVS 0.5 array configuration. The $+y$ axis points to the North and the dashed circle is $8 \mathrm{~m}$ in diameter.

\subsection{Initial tests and verification}

There are a number of questions we would like answered with the AAVSs and AAVS 0.5 in particular which include: how well does our electromagnetic (EM) simulation software predict the measured performance?; what non-idealities are introduced in practice that are not accounted for in simulations?; and can the SKALAs operate without a metallic ground plane? To begin answering these questions, however, data need to first be reliably collected and therefore the initial question must be: what are the requirements for the receiving system?

At a very basic level, a radio astronomy receiving system must be sensitive to the low-level signal it intends to receive and resistant to high-level signals it picks up in the process, i.e. it must have sufficient dynamic range. For SKA-low, the low-end of the dynamic range is not hard to determine: the system noise temperature $\left(\mathrm{T}_{\text {sys }}\right)$ should be a small fraction of the sky noise $\left(\mathrm{T}_{\text {sky }}\right)$ plus a deemed acceptable receiver noise [9]. The high-end, however, is typically limited by total sky noise in the receiver bandwidth plus unwanted radio frequency interference (RFI), the latter being often dominant and, as it is unknown for the SKALA, must be measured on-site.

Hence, we began by conducting an RFI survey of the site using a single SKALA element. Based on this information, we specified and assembled a receiver for the 16 element array to perform Galactic driftscan measurements. These results are then compared with a simulated counterpart involving sky and antenna models, thus shedding initial light on the questions outlined earlier.

\section{MEASUREMENT RESULTS}

\subsection{RFI measurement}

We performed scans from $2 \mathrm{MHz}$ to $2 \mathrm{GHz}$ with a single SKALA connected to an RFI receiver (Rohde \& Schwarz ESU 26). Fig. 3 reports in-band (70-450 $\mathrm{MHz}$ ) interferers as well as dominant interferers found in the entire frequency sweep (most notably seen in the 5 to $25 \mathrm{MHz}$ band). To first approximation, this high-level spectrum is that which each AAVS 0.5 receiver input "sees"; the information regarding receiver high-end dynamic range is contained therein.

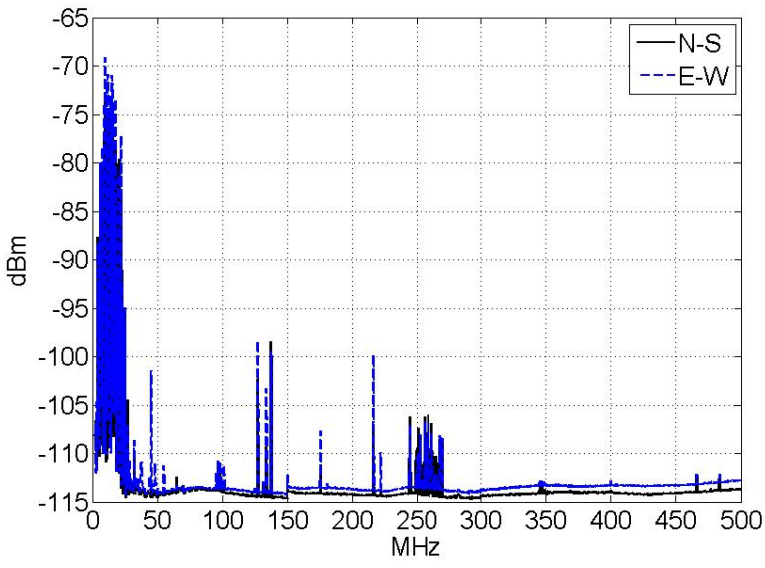

Figure 3: 99\%-tile power received by a single SKALA based on $24 \mathrm{hr}$. RFI scans (13-14 Feb. 2013); measurement settings: $\mathrm{RBW}=100 \mathrm{kHz}$; dual polarizations, N-S and E-W; 10 frequency sweeps per minute; noise floor $<-111 \mathrm{dBm}$; and dynamic range $70 \mathrm{~dB}$. The highest interferers are in the 5 to $25 \mathrm{MHz}$ band and are most likely off-shore over-the-horizon in origin.

Through a simple process of interpretation (based on continuous wave [CW] signal approximation)*, we determined that the low-frequency ( 5 to $25 \mathrm{MHz}$ ) interferers amounted to at most $-56 \mathrm{dBm}$ peak power. In comparison to other interferers, this is the only RFI band with peak power that rises above the total sky noise (approx. $-86 \mathrm{dBm}$ in the $70-450 \mathrm{MHz}$ band); hence, while in the $70-450 \mathrm{MHz}$ band the high-end dynamic range is sky noise dominated, for frequencies less than $30 \mathrm{MHz}$ it is RFI dominated. Consequently, more stringent high-end dynamic range specification is required for the low frequencies. 


\subsection{Galactic drift-scan}

Information gleaned from the RFI measurement was incorporated into a set of receiver specifications for the next phase of AAVS 0.5 measurement: Galactic drift-scans. The purpose here is to form an array beam which is excited by Galactic noise; the results may then be compared with an EM simulation of the array excited by a sky model. In addition, as all 16 elements are active in this test, the results will further refine the high-end dynamic range requirement for future AAVS 0.5 receivers.

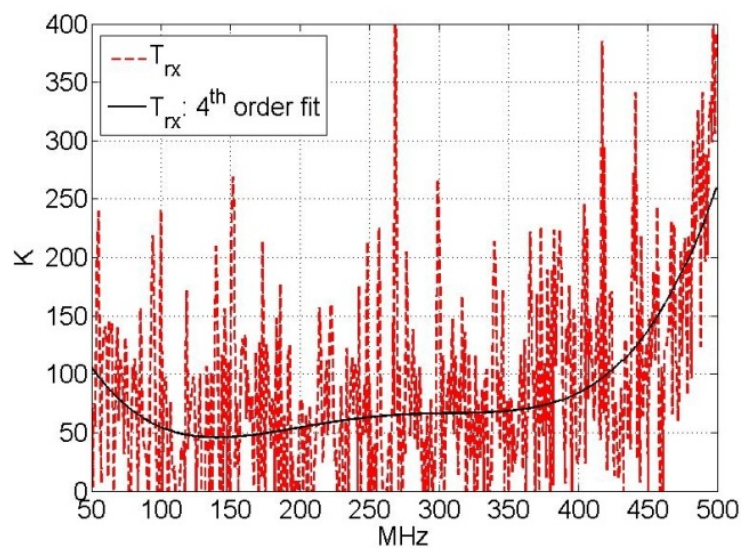

Figure 4: Measured noise temperature (using the

Hot/Cold method with averaging time of one to two minutes) of the receiver for Galactic drift-scan

measurement. The black line is a fourth-order leastsquare fit to the measurement data.

The receiver system consists of low-noise amplifiers (LNAs) connected directly to the antenna feeds [3], $15 \mathrm{~m}$ long coaxial cables (LMR195 Ultra) and an MWA beamformer modified to conform to the receiver specification. Data were recorded by an Agilent N9344C handheld spectrum analyzer. Fig. 4 reports the noise temperature of the receiver; with sky noise models in mind $\left(\sim 60 \lambda^{2.55} \mathrm{~K}\right)$, we note that the receiver is sky noise dominated to approximately 200 MHz.

Fig. 5 reports results from our measurement and modeling at $50 \mathrm{MHz}$. The AAVS 0.5 was modeled on infinite soil using FEKO (see gain pattern in Fig. 6) and excited by a well-known sky model [10]. At this frequency, the agreement is very good with the peak (04:00, Galactic center), trough (13:00, cold sky) and secondary peak (18:00, Galactic plane) evident and coincident in both plots. This is an encouraging result as it suggests two things: our system indeed measures the sky and the antenna model is at least approximately correct.

However, with increasing frequency* we noted deterioration in the agreement as the measured peak diminishes and shifts to later than 04:00. In addition, the secondary Galactic plane peak no longer appears.
This trend led us to suspect that the array might have not been phased correctly at the higher frequency which suggests the existence of a delay spread amongst the pre-summed components in the array. It prompted us to carefully characterize the delays of the said components: LNAs, cables, and beamformer. We found based on measurements of 18 cables that, at \pm 2 ns group delay standard deviation $( \pm 220$ degrees at $300 \mathrm{MHz}$ ), the nominally length-matched $15 \mathrm{~m}$ coaxial cables are the dominant source of error. The levels of errors measured are consistent with the lack of agreement between measurement and model at higher frequencies. This led us to address the problem by considering phased matched cables for subsequent deployments. Although increasing the cable preparation cost may be acceptable for the low production volume AAVS 0.5, critical tradeoff analyses are needed for AAVS 1 and beyond. These questions involve higher level system considerations such as beamforming method, array size requirement and signal transport strategies.

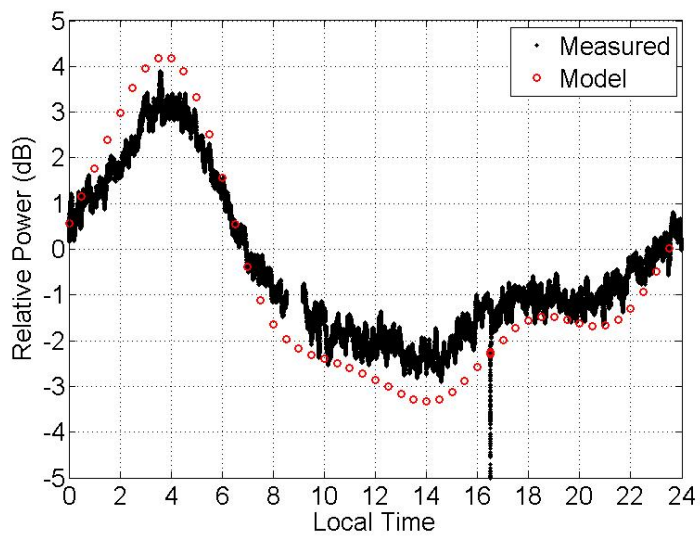

Figure 5: Measured and modeled galactic drift scan results obtained from 23 (16:30) to 24 (16:30) Apr. 2013 at $50 \mathrm{MHz}$. The $0 \mathrm{~dB}$ line indicates mean value over the $24 \mathrm{hr}$. period. Measured data was recorded every second; running average values with a $5 \mathrm{~min}$. window are plotted.

\section{NEAR-FUTURE PLAN}

We have demonstrated that simple stand-alone array tests, such as Galactic drift-scans, are of diagnostic value as they detect non-idealities and potential issues early. However, given its small size and low frequency of operation, AAVS 0.5 on its own will be largely dominated by Galactic (sky) noise, making observation of other celestial sources impractical. Cross-correlation with the MWA offers the longer interferometric baselines required to suppress the Galactic noise allowing observation of "bright" point sources from which antenna pattern and system sensitivity may be more accurately inferred and key 
design questions, including those relating to ground plane requirements, better answered. Preparations are currently underway to connect AAVS 0.5 to the MWA system and to perform the tests mentioned*.

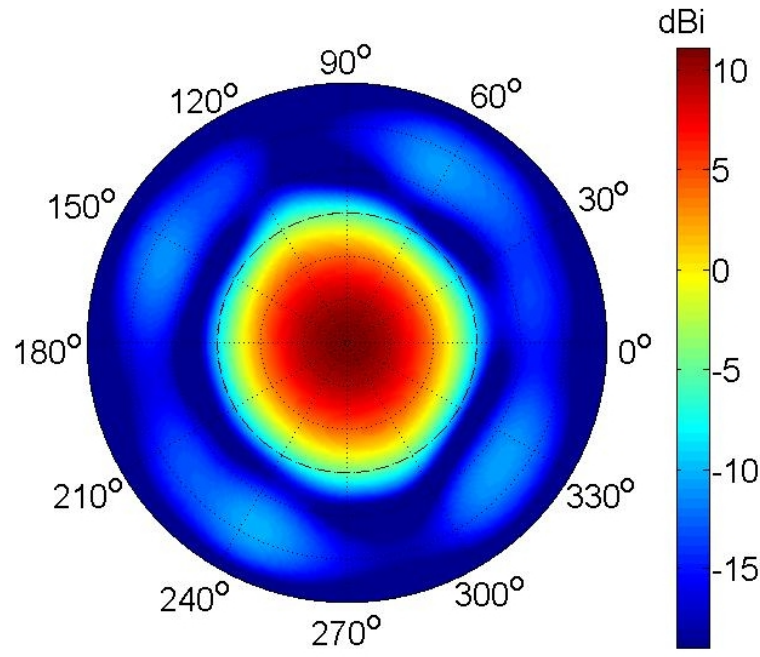

Figure 6: FEKO simulated antenna gain pattern of the AAVS 0.5 summed array at $50 \mathrm{MHz}$. The radial scale is from zenith to horizon $\left(\theta=0\right.$ to $\left.90^{\circ}\right)$. The dashed circle denotes $\theta=45^{\circ}$.

\section{CONCLUSION}

Verification systems form an essential part in the development of next-generation, large-scale systems such as SKA-low. Our early experience illustrates that seemingly trivial components such as coaxial cables could be a source of dominant error in some array architectures. As cable properties are often assumed to be ideal (or, more likely, excluded altogether) in EM simulations or system calculations, this type of finding seems unlikely without an earnest effort in prototyping.

With more advanced verification tests, including correlation with the MWA, we expect to similarly find more "trivial-but-critical" issues that challenge our assumptions. We have also learned that problems with measurement systems must be rooted out before the reliability of our EM simulations may be ascertained.

\section{Acknowledgments}

We acknowledge the Wajarri Yamatji people as the traditional owners of the Murchison Radio Astronomy Observatory site. The MRO is operated by CSIRO, whose assistance we acknowledge. The AAVS 0.5 operates as an external instrument of the MWA telescope and we thank the MWA project and personnel for their support. The authors also thank the following individuals: M. Sokolowski for the sky model, S. Padhi for the FEKO model and F. Schlagenhaufer for the RFI data.

\section{References}

[1] (2013) SKA1 system baseline design. Online. SKA Project Development Office. Manchester, UK. [Online]. Available: http://www.skatelescope.org/publications/requestfor-proposals/

[2] (2010) SKA phase 1: Preliminary system description. Online. SKA Project Development Office. Manchester, UK. [Online]. Available: http://www.skatelescope.org/publications/

[3] E. de Lera Acedo, "SKALA: A log-periodic antenna for the SKA," in Electromagnetics in Advanced Applications (ICEAA), 2012 International Conference on, sept. 2012, pp. $353-356$.

[4] E. de Lera Acedo, et al., SKA AA-low Front-End Developments (At Cambridge University), in Proc. of IEEE European Conference on Antennas and Propagation, EuCAP, Prague, mar. 2012.

[5] C. Lonsdale, et al., "The murchison widefield array: Design overview," Proceedings of the IEEE, vol. 97 , no. 8 , pp. $1497-1506$, aug. 2009.

[6] S. J. Tingay, et al., "The Murchison Widefield Array: the Square Kilometre Array Precursor at low radio frequencies," Publications of the Astronomical Society of Australia, 30, e007 doi:10.1017/pasa.2012.007.

[7] Y. Lo, "A mathematical theory of antenna arrays with randomly spaced elements," Antennas and Propagation, IEEE Transactions on, vol. 12, no. 3, pp. $257-268$, may 1964.

[8] W. van Cappellen, S. Wijnholds, and J. Bregman, "Sparse antenna array configurations in large aperture synthesis radio telescopes," in Radar Conference, 2006. EuRAD 2006. 3rd European, sept. 2006, pp. $76-79$.

[9] W. A. Van Cappellen, J.D. Bregman and M. J. Arts, "Effective sensitivity of a non-uniform phased array of short dipoles," Experimental Astronomy (2004) 17: 101-109

[10] A. De Oliveira-Costa, M. Tegmark, B. M. Gaensler, J. Jonas, T. L. Landecker and Patricia Reich, "A model of diffuse Galactic radio emission from $10 \mathrm{MHz}$ to $100 \mathrm{GHz}$," MNRAS (2008) 388 (1): 247-260. doi: 10.1111/j.1365-2966.2008.13376.x 